\title{
On the Effectiveness of Obligations of Labor Standard Law to Employment Contract
}

\author{
Feng Pan \\ School of Law, Xiamen University, Xiamen 361005, China \\ xmupanfeng@vip.163.com
}

\begin{abstract}
None of the Theory of Reflection Effect, the Theory of Direct Private Law into Effect, and the Theory of Direct Effect in the Third Scope of Law can provide appropriate theoretical explanations for the contract validity of obligations under labor standard laws of China. The labor standard laws of China bear the weight of functions to directly adjust rights and obligations relations under private laws on labor relations subject, with the nature of public laws mixed with private laws. The purposes under labor standard laws is of difference, which can be fallen into pure obligation under public laws and those with double attributes of both public laws and private laws. Only obligations under labor standard laws with double attributes of both public laws and private laws have most direct effectiveness of private laws, and supplement and replace contract content. The effectiveness produces directly on the basis of the labor standard laws and norms, not through the transform of the general terms and conditions of employment contract laws.
\end{abstract}

Keywords: labor standard law; employment contract; labor relation; private law

\section{论劳动基准法义务的契约效力}

\author{
潘峰 \\ 厦门大学法学院, 厦门 中国
}

摘 要: 反射效力说、直接私法转化效力说、第三法域直接效力说都不能为中国劳动基准法 义务的契约效力提供适当的理论解说。中国劳动基准法承载了直接调整劳动关系主体私法上 权利义务关系的功能, 具有公私法混合的性质。劳动基准法义务依规范目的的不同, 可分为 纯粹的公法义务和兼具公法与私法双重属性的义务。只有兼具公法与私法双重属性的劳动基 准法义务才具有直接的私法效力, 补充和替代合同的内容。这一效力直接依据劳动基准法规 范产生，无须通过劳动合同法的一般条款进行转化。

关键词: 劳动基准法; 劳动合同; 劳动关系; 私法

\section{1. 前言}

劳动法是一个富于变化的多重结构的法律领域, 其规范分为各种不同的层次, 且分散在 不同的法律法规中, 结构较民法更为复杂。从宏观角度分析, 劳动法的体系结构可划分为劳 动关系协调法、劳动基准法和劳动保障法三大类。劳动合同法属于劳动关系协调法的范畴, 而劳动基准法则由实现劳动条件基准化为基本职能的各项劳动法律制度所构成，包括工资、 工时、劳动保护、劳动监督等法律。基于劳动合同法的一般条款和具体权利义务条款, 当事 人之间发生以提供劳务和支付工资为内容的主给付义务, 以及依诚实信用原则所产生的附随 义务。同时, 依据劳动基准法规范, 国家以劳动基准作为劳动条件的上限或下限, 强制雇主 承担一定的作为义务或不作为义务, 并通过有关国家机关的行政处罚甚至刑罚制裁等方式以 确保这些义务得以履行。那么, 劳动基准法义务对劳动合同关系有何影响? 劳动基准法义务 与劳动合同义务之间有何关联? 直到目前为止, 中国大陆学者尚未对这些问题作出有力的理 
论说明。而这些问题的研究, 又直接关系到劳动基准法对劳动者契约权利的直接保障抑或间 接保障, 有必要加以讨论, 以弥补劳动法基本理论的缺漏。

\section{2. 既有学说之梳理}

关于劳动基准法义务对劳动合同的效力问题, 理论上迄今未有统一的认识, 主要有三种 学说。其中, 反射效力说为中国台湾地区理论界通说, 直接私法转化效力说由黄程贯教授自 德国法学理论引介而来, 第三法域直接效力说则产生自中国社会法学研究土壤, 可以看作是 理论本土化的产物。三种学说虽对劳动基准法义务的契约效力来源存在争议, 但都认为, 在 雇主违反劳动基准法义务时, 劳动者可以主张私法上的权利救济。

2.1. 反射效力说

反射效力说以劳动基准法属于公法作为理论出发点, 认为劳动基准法调整的是国家与雇 主间的关系, 劳动者仅为权利义务关系的受益人, 而非权利主体。中国台湾地区学者林丰宾 先生认为, 劳动基准法以保护劳动者为其主要目的, 但其保护并非本于雇主与劳动者之间所 订立的劳动契约, 而是以整个社会经济和国家公共利益为其出发点, 是雇主与国家间的公法 关系。换言之, 劳动保护义务不是私法上契约的义务, 而是公法上雇主对于国家所负的义务。 因此, 就其性质而言, 劳动基准法并非直接规定雇主与劳动者之权利义务关系, 而系规定国 家与雇主间之权利义务关系。所以劳动基准法强制雇主应履行的义务, 乃是以国家为权利人, 而劳动者仅因其为雇主义务履行的对象而受益, 亦即所谓 “反射利益”。 ${ }^{[1]}$ 既然劳动者不属 于劳动基准法律关系的权利人，自然无权抛弃权利，也无权免除劳动基准法上所规定的雇主 义务。因而, 如果雇主未依照劳动基准法履行义务, 请求权利人是国家, 而非劳动者; 国家 为达成其强制的目的, 采取行政手段和刑事手段对雇主施加惩罚。那么, 劳动者如何依据劳 动基准法获得私法救济? 反射效力说对此采取了一种迂回的策略, 认为劳动基准法规范构成 私法上的强制禁止规定, 违反时会产生行为无效的法律后果, 亦可将之认定为侵权行为法上 所谓的 “保护他人之法律” ，违反时即推定行为人有过失，而须负损害赔偿责任。通过此等 途径, 公法性质的劳动基准法即在私法上产生其作用与效力。

2.2. 直接私法转化效力说

直接私法转化效力说认同劳动基准法的公法性质，但其主张与反射效力说不同之处在 于, 劳动基准法在产生雇主对国家所承担的公法义务的同时, 也通过民法关于雇主保护义务 的法律机制, 形成相同内容的私法契约义务。德国法学界通说认为, 劳动基准法对雇主所课 加的强制性公法义务，会内容一致地形成雇主对劳动者的强行法性质的契约义务。 ${ }^{[2]}$ 不过， 根据直接私法转化效力说, 劳动基准法规范对契约义务内容的形成效果并非其本身所产生, 也非借由法律解释所能导出, 而是须有私法上的转化根据, 而此根据即是民法中关于雇主对 劳动者所负保护义务的一般条款或劳动契约章节的相关规定。至于所转化的义务类型, 则视 该义务所涉及的利益而定。由于劳动者被纳入雇主的指挥管理范围内提供劳务, 所以雇主对 劳动者所可能产生的各种危害即负有强化的保护照顾义务, 此义务乃是由诚信原则所导出, 原则上系契约的附随义务。但若劳动基准法义务所保护的内容涉及劳动者生命、健康等重大 利益, 则此时所转化成的契约义务即为雇主的主给付义务, 而非仅是附随义务的层次。

2.3. 第三法域直接效力说

第三法域直接效力说彻底抛弃了劳动基准法属于公法的观念，认为作为社会法的劳动基 准法是公私法融合的法律，其义务主体和法律责任都不同于传统的私法或公法规范，应跳出 公法或私法的范畴内理解。第三法域直接效力说是中国大陆某些学者在研究社会法基本理论 的基础上提出的, 目前虽未有直接的理论阐释, 但从董保华教授等人的著述中可以归纳出这 一观点。第三法域理论认为, 社会法不具有传统的公法或者私法的典型性质, 而具有公法和 私法融合的特征。考虑到此种融合的复杂性, 拘泥于传统的公私法理论难以作出说明, 最佳 的方式是为之专门划出一块相对独立的领域, 这就是第三法域。在第三法域, 国家利用 “有 形之手” 来矫正市场机制的内在缺陷, 在市场失灵时, 越过 “公域” 的界限, 介入 “私域” , 
突破了传统法律部门之间旧有的疆界, 将不同的法律规范、尤其是从古典乃至近代的私法规 范中引申出来的各种原理重新整合, 不仅顾及权利人的利益, 而且强调与义务人的关联。劳动 基准法义务对劳动合同直接发生效力, 乃是第三法域独特效力的体现, 无须再对公法和私法 作出界分。劳动基准法的强制性规范所产生的权利义务有类似行政权力的特点。国家从保护 社会利益的立场出发, 将一些劳动者的利益不仅规定为用人单位的义务, 也规定为劳动者的 义务。 ${ }^{[3]}$ 劳动者作为受益人, 并不是权利人, 不能随意改变或放弃自己的利益。雇主所承担的 义务是对社会的义务, 即所谓公法上的义务。 ${ }^{[4]}$ 在劳动基准法被违反的情况下, 国家机关是 权力人, 公权力通过行政程序直接介入。劳动者作为利益人虽只是义务人, 但也可以通过举 报，促使公权力介入。由于这时国家机关也是义务人，对于国家机关的不作为，劳动者可以 以受益主体的身份成为行政相对人，而提起行政诉讼。

\section{3. 劳动基准法的混合性质}

劳动基准法是具有公私法混合属性的法律。首先, 单纯的公法或单纯的私法难以调整劳 动关系, 劳动法兼有公法和私法的双重属性。雇主与劳动者之间, 一方提供劳动力, 一方支 付工资报酬, 就其形式而言, 本属于私法的雇佣关系范畴, 但其影响的层面已逾越单纯的劳 务和报酬关系, 具有经济的、社会的以及人性的实质意义。故而, 除了劳资双方的私法关系 以外, 国家公权力积极介入与干预, 形成复杂而多层面的法律关系, 此即劳动关系的社会化。 劳动法除了规范劳动关系主体的私法权利义务外, 还要求国家为社会公共利益的需要有效规 制劳动关系, 对劳动者的生存权和个人尊严予以关注和提供法律保障, 并作为法定义务要求 国家通过调整社会分配承担起国家责任。其次, 在劳动法律体系内部, 劳动合同法与劳动基 准法的属性虽有所不同, 但都是公法规范和私法规范的结合体, 并不能断言它们属于纯粹的 公法或私法。中国学者一般认为, 劳动合同法是劳动法私法性质的主要体现, 而劳动基准法 则是公法性质的集中展现。不过, 公法规范和私法规范在劳动立法中往往交融和揉杂在一起, 很难进行截然明确的划分。就劳动合同法而言, 合同化是第一位的, 私法规范占主要部分。 对于劳动基准法来说, 国家通过预设工资、工时和劳动保护等劳动基准对劳动关系进行强制 性干预, 以法律形式保障劳动者获得人格尊严及合理的生存条件。虽然劳动基准具有普适性 和基础性, 针对的是劳动者群体, 但同时其保护的对象也包括劳动者个体。换言之, 劳动基 准法规范以促进劳动者群体的公益为目的, 但同时也为个别劳动者的私益而存在, 其公法属 性与私法属性实为一体之两面。国家通过行政处罚和刑事制裁的手段强制雇主履行公法义 务，同时也以私法手段保障个别劳动者的契约权利得以实现。

而且, 对于劳动基准法性质的讨论应当在各国劳动立法自身的独特语境下展开。中国不 具有大陆法系国家将民法作为劳动合同基础性法律的传统, 中国劳动基准法承载了直接调整 劳动关系双方当事人私法上权利义务关系的功能。2007年《劳动合同法》尚未出台以前, 雇 主对劳动者的义务通过《劳动法》和大量的劳动基准立法进行规制。劳动基准法是国家对劳 动关系实行多元化调整的产物, 除了规定国家与雇主间的权力-服从关系以外, 雇主与劳动者 之间的私法权利义务亦由劳动基准法调整。实务中，劳动者也往往依据大量的劳动基准法规 范直接主张自己的私法权利。即使在《劳动合同法》实施后, 劳动基准法仍然具有同时调整 国家、雇主、劳动者三方之间关系的性格。第一，尽管《劳动合同法》以一般条款和诸多具 体条款设置了雇主与劳动者的契约义务, 但劳动关系复杂和多样性的特点决定了《劳动合同 法》难以单独实现劳动关系主体利益分配的功能。《劳动合同法》第三章 “劳动合同的履行 和变更” 除了在第29条对合同履行做出一般性规定, 第30条和第31条对雇主履行工资给付义 务加以规定之外, 对雇主与劳动者在合同履行期间的义务基本上付之成如。林嘉教授指出, 法律规范中的行为模式如果不明确, 将导致行为人无所适从。由于合同权利义务的相对性, 即双务合同一方当事人的权利总是对应另一方当事人的义务, 在合同中规定义务更为明确, 而且更容易与后面的法律责任建立逻辑上的联系。 ${ }^{[5]}$ 对于《劳动合同法》的遗漏, 我们只能 通过适用劳动基准法的义务性规范加以补充。第二, 中国劳动法学界一种有代表性的观点认 
为, 劳动法与民法是两个并行而独立的领域, 当劳动合同没有规定时不能适用合同法的一般 原理，而应适用劳动法的基本原则和法理。 ${ }^{[6]}$ 民法上的义务分配规则不能为劳动合同所当然 吸收时, 对于缺失的义务规范, 除了根据《劳动合同法》一般条款推导之外, 求助于劳动基 准法, 直接认可其义务规范对劳动契约发生效力是极为必要的。第三, 中国部分劳动基准立 法在规定了义务及违法应承担的公法责任之外, 有时还设置了雇主违法行为的私法责任, 这 也可以看作是中国劳动基准法具有混合属性的佐证。

那么, 既然劳动基准法同时包含公法和私法的规范或者说具备了公私法融合的特征, 采 用 “第三法域直接效力说” 解说劳动基准法义务的效力是否合理? 本文认为, 我们仍应从 公法或者私法的角度来划分劳动基准法规范的效力, 而不可简单地将其划入公法或者私法之 外的第三法域。劳动基准法作为社会法的组成部分, 基于保护劳动者利益的需要, 虽然在一 定程度上突破了严格的公私二元划分, 更为精细地调整社会生活, 但并未因此根本颠覆公、 私法划分的基础, 其性质依然可以纳入公私法二元结构中加以理解。部分劳动基准法规范同 时具备公私法属性，具有不同于传统公法或私法规范的特色。如果我们对劳动基准法规范的 公法效力和私法效力不加以区分, 过多地强调劳动法相对于民法的异质性, 很可能不适当地 扩张公法对劳动者的保护, 而忽略了劳动者私法上请求权的实现。

\section{4. 劳动基准法义务对劳动合同的直接效力}

劳动基准法的混合性质决定了其义务可能同时发生公法和私法上的双重效力。当然，也 不是说劳动基准法中所有的义务规范都具有直接调整私法关系的功能。劳动基准法义务对劳 动合同产生直接效力, 前提是该义务的内容适于成为劳动合同的义务内容。为此, 我们必须 对不同类型的劳动基准法义务做出识别。劳动基准法义务依其规范目的的不同, 可分为以下 两类:

首先是纯粹的公法义务。此种义务基于国家对社会利益的保护而产生，仅调整国家与雇 主间的法律关系, 劳动者为受益主体而非权利主体。劳动基准法对此种义务的规范目的在于 国家机关监督或管理上的方便或取得信息, 仅具有组织的或规范法上的功能, 或者以全体的 劳动者作为保护对象, 不在于直接保护个别劳动者, 属于完全的公法义务, 劳动者不能以此 作为对雇主的请求权基础。例如，《安全生产法》第18条规定：“生产经营单位应当具备的 安全生产条件所必需的资金投入，由生产经营单位的决策机构、主要负责人或者个人经营的 投资人予以保证，并对由于安全生产所必需的资金投入不足导致的后果承担责任。”这一规 定的根本目的虽在于保护劳动者的人身安全, 但从规范的直接功能而言, 仅涉及国家与雇主 间的权力-服从关系，使得国家能够运用行政手段或刑事手段对雇主违反义务的行为加以监 督, 不在于保护个别的劳动者, 不适于作为劳动合同的内容。当雇主违反此规范时, 劳动者 也不能以此作为请求权基础。在纯粹的公法义务被违反, 劳动者的利益受侵害的情况下, 劳 动行政部门应依法行使职权, 公权力可以主动通过行政程序介入。劳动者作为受益人, 也可 以举报、投诉的方式, 促使公权力介入。如果行政机关不作为, 劳动者可以受益主体的身份, 提起行政诉公。

其次是兼具公法与私法双重属性的义务。此类义务虽然规定在劳动基准法中，但其规范 目的在于直接保护个别的劳动者, 可以将之视为劳动合同的义务。在此种场合, 劳动基准法 规范具有公私法的双重属性，同时发生公私法上的效力。例如，2001年《职业病防治法》第 32 条第 1 款规定： “对从事接触职业病危害的作业的劳动者, 用人单位应当按照国务院卫生 行政部门的规定组织上岗前、在岗期间和离岗时的职业健康检查, 并将检查结果如实告知劳 动者。职业健康检查费用由用人单位承担。”依此规定, 一方面雇主被课以公法上的义务, 一旦违反将受到行政处罚：由卫生行政部门责令限期改正，给予警告，可以并处 2 万元以上 5 万元以下的罚款; 另一方面, 此义务也当然成为劳动契约的内容, 雇主应尽到职业健康检查 的劳动合同义务, 劳动者可要求雇主履行。 


\section{5. 结论}

只有兼具公法与私法双重属性的劳动基准法义务才具有直接的私法效力。这一效力直接 依据劳动基准法规范产生, 无须通过劳动合同法的一般条款进行转化。首先，劳动基准法义 务具有补充效力。当雇主与劳动者在劳动合同中对于对应义务无约定时, 劳动基准法义务直 接成为合同义务, 补充和完善劳动合同的内容。其次, 劳动基准法义务具有替代效力。劳动 基准法义务设定了合同义务的最低标准，限制了劳资双方自由协商的空间，雇主不得向劳动 者提供低于劳动基准法规定的劳动条件。当双方约定的劳动条件低于劳动基准法要求时, 劳 动基准法义务直接替代原来的条款, 直接成为合同内容。最后, 当雇主违反劳动基准法义务 时, 劳动者可以依据劳动基准法规范对雇主主张合同上的权利。依照黄程贯教授的看法, 劳 动者所得主张的权利包括: 履行请求权、给付拒绝权、损害赔偿请求权与契约终止权。 ${ }^{[2]}$ 但 在中国大陆地区, 劳动者若要主张上述权利, 其请求权基础尚不完整。完整的法律规范由行 为模式和法律后果组成。法律责任的欠缺是中国立法的通病, 劳动基准法也不例外, 往往仅 规定了雇主的行为模式和公法后果, 未能提供相应的私法后果。劳动基准法作为直接指向当 事人权利义务的法律规范, 既然提出了对当事人私法行为规则的要求, 自然应当有相应的法 律责任的规定。因此, 应完善现行劳动基准法中的私法责任规范, 赋予劳动者完整的请求权。 否则, 仅仰赖劳动保障部门的主动介入或劳动者提起行政诉讼的方式, 均不足以保证雇主充 分履行其劳动基准法义务。

\section{致谢}

本文系中央高校基本科研业务费专项资金资助项目 “企业惩戒法律问题研究” (T2013221003) 成果之一。

\section{References}

[1]. Lin Fenbin, Labor Standard Law, (Taipei: San Min Book Co., Ltd., 2003), p.13.

[2]. Huang Chengguan, " The Public Law Nature of Labor Standard Law and its transformation to private law " Soochow Law Review, Vol.12, 2006, pp.8-25.

[3]. Dong Baohua, Social Law Theory,(Beijing: China University of Political Science and Law Press, 2001),p.185.

[4]. Dong Baohua, Zheng Shaohua, "Social Law : Exploration of the third law field " Journal of East China University of Political Science and Law, Vol.12, 1999 No. 1, pp.30-37.

[5]. Lin Jia, Yang Fei, " Discussion on Legal Issues in the Legislation of Employment contract " Study and Exploration, Vol.38, 2006 No. 5, pp.98-102.

[6]. Lin Jia, "Study on Legal Issues in Labor Contract "the Jurist, Vol.14, 2003 No. 6, pp.65-72. 